\title{
Car following and lane changing behavior using NGSIM and China data
}

Md. Mijanoor Rahman, Mohd. Tahir Ismail, Majid Khan Majahar Ali

School of Mathematical Sciences, Universiti Sains Malaysia, Malaysia

\begin{tabular}{|c|c|}
\hline Article Info & ABSTRACT \\
\hline Article history: & \multirow{10}{*}{$\begin{array}{l}\text { Road safety is imperative theme because increasing road fatalities deaths in } \\
\text { world. Besides road fatalities, traffic jam is increasing, human is frustrated } \\
\text { for uncomfortable journey. The roads safety and passengers comfortable of } \\
\text { the roadway system are vastly depended on the Car following (CF) and Lane } \\
\text { Changing (LC) features of drivers. CF and LC theory describe the driver } \\
\text { behavior by following paths in a traffic stream. In this research, researchers } \\
\text { have compared to US-101 Next-Generation-Simulation (NGSIM) data with } \\
\text { Beijing forth ring road, China freeways real trajectory data by CF and LC } \\
\text { models. The CF data has been calibrated with Genetic Algorithm (GA). } \\
\text { Reproducing Kernel Hilbert Space (RKHS) is generated the LC beginning } \\
\text { and finishing points. Findings revealed that the CF parameters as maximum } \\
\text { acceleration, minimum deceleration, free speed, minimum headway and } \\
\text { stopping distance percentages of Chinese data are } 74.71 \%, 79.95 \% \text {, } 66.57 \% \text {, } \\
0.018 \% \text { and } 65.65 \% \text { respectively of NGSIM data. After completing the } \\
\text { comparison, researchers have been found out optimization safety and } \\
\text { comfortable acceleration-deceleration and LC beginning-finishing points of } \\
\text { driver behavior. Here this analysis generates the driver behavior at real traffic } \\
\text { network on the express highways of specific two roads US- } 101 \text { (NGSIM) } \\
\text { data and Chinese freeways data. Since NGSIM data is well simulated so road } \\
\text { traffic is more safety and comfortable for journey. }\end{array}$} \\
\hline Received Nov 30, 2018 & \\
\hline Revised Feb 7, 2019 & \\
\hline Accepted Feb 18, 2019 & \\
\hline Keywords: & \\
\hline Calibration & \\
\hline Car following & \\
\hline Genetic algorithm & \\
\hline Lane-changing & \\
\hline Vehicle dynamics & \\
\hline
\end{tabular}

Copyright (C) 2019 Institute of Advanced Engineering and Science. All rights reserved.

\section{Corresponding Author:}

Majid Khan Majahar Ali, School of Mathematical Sciences, Universiti Sains Malaysia, 11800 USM, Penang, Malaysia. Email: majidkhanmajaharali@usm.my

\section{INTRODUCTION}

In 2017, China is global massive Automobile marker exceed USA with demand-supply and after 2020, 200 million passenger vehicles and loaded vehicles will be emerging in the traffic road network [1]. Then, not only people are increasing but also vehicles are increasing progressively. It has emanated the increased traffic exposure which has more road traffic crashes [2]. By road crashes, every year 1.25 million human lives decease, more than 1.2 million road fatalities and above 50 million peoples are sickened whole world. But every country has not same propagation traffic fatalities. Road crash is rising geometrically in every country, although developing countries like as China ensues more traffic fatalities than developed countries like USA. Road crash has been taken seriously by developed countries. Only $10 \%$ road crash ensues in these types of countries. UN also proclaimed that road safety is now thoughtful disclosure entire universe [3]. In traffic network, CF and LC are very important mater. CF and LC model are being analyzed for traffic safety and comfortable journey $[4,5]$.

A complex traffic network behavior has been analyzed different types of traffic flow models as microscopic and macroscopic. Macroscopic model is compressible vehicles runny formed. Microscopic model is vehicle discrete position and running by dissymmetry situations [4], Microscopic simulation 
produced a significant part in the exploration and plan of traffic network facilities. It delivers very flexible policy on which many traffic network situations can be deliberate in a measured operation without troublemaking real-world traffic [6,7]. Generally, a traffic network simulation platform contains of numerous models which discourse different features of traffic network behavior [8-12].

Most of the microscopic simulation accept ordinary difference equations categorized by a rare parameter to explain driver Car Following Model (CFM). Apropos promise the simulation models glowing reproduce traffic scenario detected in preparation, diverse calibration methods are proposed to fittingly control these types of model parameters [9, 10, 12-14].

A rare parameter has somatic parallels in roughly microscopic CFMs like as preferred velocity conducted in Gipps CFM [9, 15]. Farther macroscopic structures of traffic network flow parameters like as critical density, free flow velocity and jam density are significant in numerous models and can be directly projected from circle indicator data [8]. Nevertheless, in lots of CFMs, supplementary parameters can't be merely derived from macroscopic capacities [9, 16-18].

The calibration of CFMs by using observed vehicle road trajectory data enlarged interests recently, road trajectory data delivered more of driving behavior on the microscopic traffic flows [9-13, 19]. Accurately by data estimation, the model parameters are now very importance issue from real road trajectory data. Nobody could perform to perfectly fit the data with real trajectory CF data $[6,17]$.

Most efficient CFM, IDM was introduced and then it was used for given many significant rules at traffic network [6]. The IDM is a CF dynamical system by using many physical formulas of velocity, acceleration and deceleration. This IDM system give simulation data by using many parameters like as initial position, velocity, acceleration or deceleration of lag and front vehicle and gap and time headway between vehicles.

Researchers have taken very widely usable optimization algorithm GA for calibration of CF simulation data to trajectory data. There have many optimization functions for calibration of simulation data to trajectory data like as sequential-quadratic-programming-Algorithm (SQPA), simultaneous-perturbationstochastic-approximation-algorithm (SPSAA), Nelder-Mead-algorithm (NMA) and some others [20]. To find an optimal solution of parameter values was used calibration problem [15], the Nelder-Mead-algorithm $[21,22]$. GA is the most efficient and deserved very nearly accurate value for the optimization other than many optimization function [23]. Paz et al. [18] found the best CF parameter from CORSIM software data by calibration with real trajectory data [18]. They used GA and SPSAA for microscopic car following calibration method. For CF parameters calibration, a stochastically global search GA is most broadly used system for unconstrained and constrained objective functions [24]. Li et al. [20] explained about GA method surely give CF calibration parameters [21]. In this research, authors have analyzed the IDM, CFM and calibrated the parameters by Sum of Square Error (SSE) objective function using widely functioning and very efficient GA algorithm [25].

Nilsson and Sjöberg [26] worked on binary lane choice system such as one or zero, current lane is zero and change lane is one and velocity profile such as collision time and control velocity for car driver. The authors analyzed the LC maneuver by only collision time LC behavior [26]. Hongtao et al. [27] proposed multiplayer Stackelberg game-theory based LC maneuver by using safety factor, gap acceptance, position, velocity and acceleration utilities of host car and surrounding car for human driver. Here, gap acceptance is depended on safety factor, position difference and velocity difference not acceleration. The researchers nicely represented the car lateral and longitudinal position into the LC time but didn't calculated the optimization LC position of the car. A human driver could maintain the LC maneuver without any wireless connection among the drivers only using signal light. The optimization LC starting (time and position) and ending (time and position) and could be calculated in their article [27]. Marinho et al. [28] discovered search algorithm RKHS, non-parametric regression fitted system for analyzing real trajectory data sets [28]. C. Dong et al [29] proposed new LC model using reproducing kernel Hilbert space (RKHS) for optimizing the LC beginning and finishing point without collision. RKHS is very efficient non-parametric regression analysis is used on trajectory LC maneuver with optimum distance function [29]. The scientist worked on US 101 NGSIM real trajectory data for finding the beginning and finishing time. On LC time, the beginning and finishing time is very important for the driver because every driver want to avoid collision with other driver for safety and but worked on only real trajectory data not any specific model or maneuver. In this research, authors have analyzed the gap acceptance LCM and beginning and finishing points of LC maneuver using RKHS by NGSIM data and Chinese data. This work is bearing to develop a model of driver CF and LC in the expressed highway with on-ramp and off-ramp and urban congested traffic area. 


\section{METHODOLOGY}

The detail methodology of CF with calibration and LCMs are analyzed in this section. IDM CFM generates the simulation data, this simulation data calibrates the IDM parameter by SSE objective function with GA algorithm. After that, in methodology is given the LC behavior of driver by using RKHS, nonparametric regression fitted model from NGSIM data. In this research, not only are the nearest ego and following vehicles in the existing and neighboring lanes considered but also the closer ego and following vehicles. The different car following and lane changing model has been developed by using MATLAB coding and extract trajectory road traffic data from Beijing forth ring road, China and US-101, NGSIM data. These models have been simulated about known parameters then calibrated according the trajectory data. These systems are discussed in the following.

\subsection{CFM}

In traffic-flow modeling, the Intelligent-Driver-Model (IDM) is a microscopic traffic-flow model for simulation of urban and freeway traffic. This model has been discussed as follows $[9,30]$ :

Distance between ego car $n$ and lag car $(n-1)$ is

$$
s_{n}=x_{n-1}-x_{n}-l_{n-1}
$$

Where $x_{n}, x_{n-1}, l_{n-1}$ denote the position of ego car $n$ and lag car $(n-1)$ and length lag car $(n-1)$ respectively.

$$
\Delta \dot{x}=\dot{x}_{n}-\dot{x}_{n-1}
$$

Where $\dot{x}_{n}$ and $\dot{x}_{n-1}$ are the velocity of the $n-t h$ car and $(n-1)-t h$ respectively.

The simplified version of IDM model is follows

$$
\ddot{x}_{n}=a\left[1-\left(\frac{\dot{x}_{n}}{\dot{x}_{0}}\right)^{\delta}-\left(\frac{s^{*}\left(\dot{x}_{n}, \Delta \dot{x}_{n}\right)}{s_{n}}\right)^{2}\right]
$$

Where the desired gap $s^{*}$ is defined by

$$
s^{*}\left(\dot{x}_{n}, \Delta \dot{x}_{n}\right)=s_{o}+\max \left[o,\left(\dot{x}_{n} T+\frac{\dot{x}_{n} \Delta \dot{x}_{n}}{2 \sqrt{a b}}\right)\right]
$$

Where $1-\left(\frac{\dot{x}_{n}}{\dot{x}_{0}}\right)^{\delta}$ is the desired acceleration for ego vehicle and is $\left(\frac{s^{*}\left(\dot{x}_{n}, \Delta \dot{x}_{n}\right)}{s_{n}}\right)^{2}$ is the braking deceleration for front vehicle. The following parameters values of IDM CFM have been explained [21]:

regular acceleration, $a=1.4 \mathrm{~m} / \mathrm{s}^{2}$,

minimum deceleration, $b=2.0 \mathrm{~m} / \mathrm{s}^{2}$,

desired speed, $v_{0}=30 \mathrm{~m} / \mathrm{s}$,

desired headway, $T=1.5 \mathrm{~s}$,

jam minimum distance, $s_{0}=2 \mathrm{~m}$ and

acceleration exponent, $\delta$

The CF simulation flowchart as follows in Figure 1.

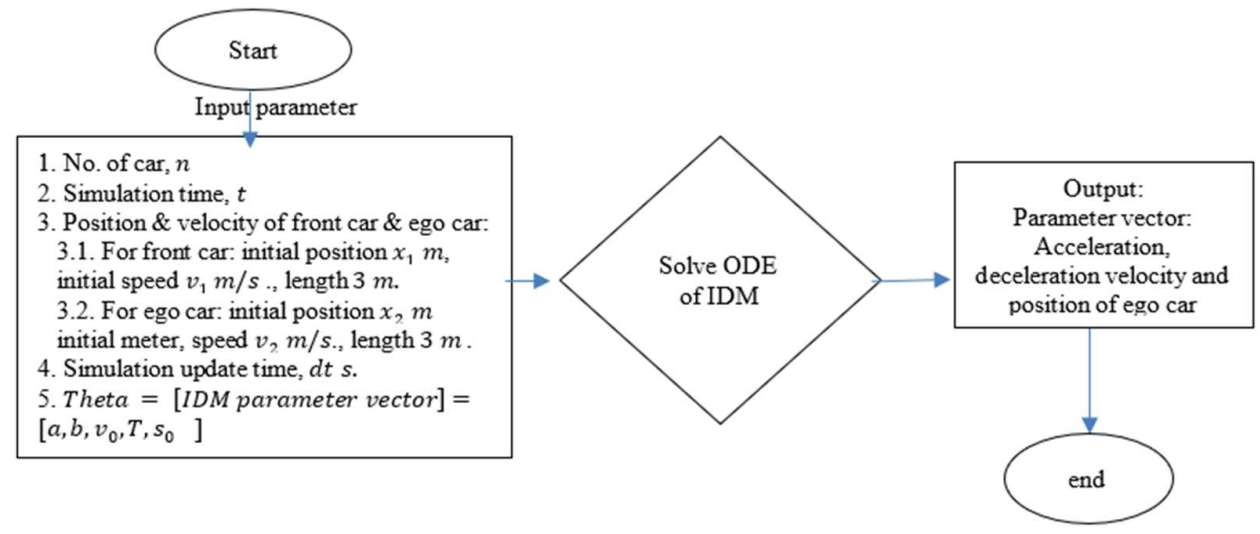

Figure 1. CFM simulation flowchart

Int. J. of Adv. in Appl. Sci. Vol. 8, No. 1, March 2019: 14 - 25 


\subsubsection{Calibration process}

Calibration for CF give the better result for simulation $n$ number of car data with compared to the trajectory same number of car data. This comparison is measured of headway, gap, velocity and acceleration by optimization objective function [11]. Researchers have analyzed well known (SSE) function of velocity and position for calibration between simulations $n$ number of car data with compared to the trajectory same number of car data [21]. Ciuffo and Punzo [31] worked on parameter calibration of synthetic data with AIMSIM software data using some Goodness of Fit (GoF) as (SSE), Root Mean Square error (RMSE) and Normalized Root Mean Square error (RMSN) to objective function (OF). SSE is most suitable and widely used best GoF for car following calibration method than other GoF [23, 31].

$$
\operatorname{Min}_{\theta \varepsilon \Omega}=\sum_{i=1}^{N}\left[v_{n}(i T \mid \theta)-\hat{v}_{n}(i T)\right]^{2}
$$

Where $\Omega$ is optimization value of solution space $\theta, v_{n}(i T \mid \theta)$ and $\hat{v}_{n}(i T)$ denote the simulated and empirical position or velocities of the $n-t h$ CF at time $t=i T$, where $=2,3, \ldots, N$, respectively. $\hat{v}_{n}(i T)$ is taken as known inputs (real trajectory data) in the optimization problem.

Decision variable $\theta$ is not to easily applicable in the optimization function and $v_{n}(i T \mid \theta)$ is depended to the (5). Here, the derivative variable of this optimization objective function is difficult to achieve the value. For overcome this condition, Researchers have analyzed the Genetic Algorithm (GA) optimization procedure for finding the objective value.

\subsubsection{Genetic algorithm (GA)}

In the research, GA chooses to save appropriate chromosomes and remove weak chromosomes from parent chromosome (PC) grade. Every simulation time, GA can update of its parent's value then compare to the previous values and after validating for next parents. The optimization procedure GA works as follows: randomly takes some simulation values (chromosomes) that are parent chromosomes (PCs) and finds the feasible values by (5). Then it deletes some digit (chromosome) from highest some efficient value and interchange their digit for find two new values. And it compares to the previous values then it is new parents. Again, find the feasible value from (5). If desired optimization value gain, then it is stopped other than the system continues. By the above way, GA can check then update every value by change the chromosomes at every iteration for desired optimization values. The Genetic Algorithm (GA) flowchart as shown in Figure 2.

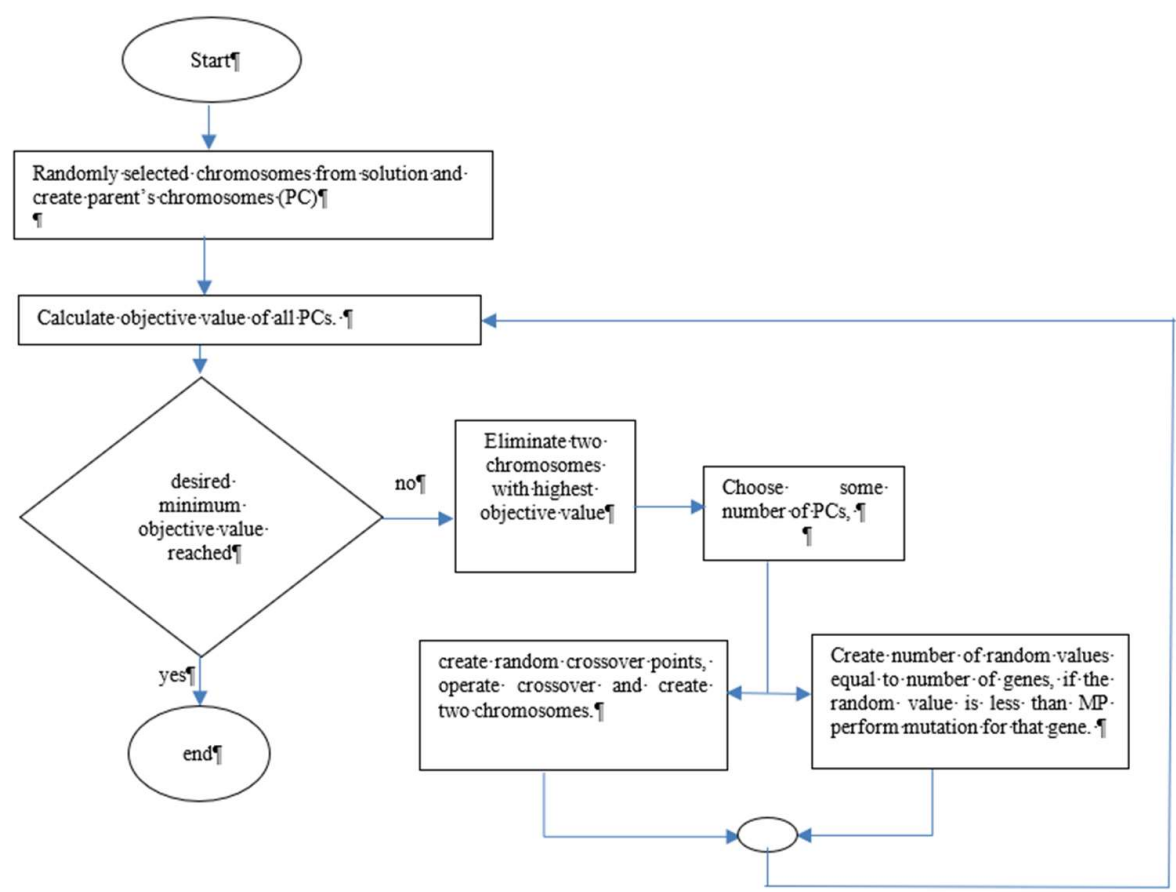

Figure 2. Genetic Algorithm (GA) Flowchart 


\subsection{LC model}

Again, conditional on the drive, LC decision is characterized by two maters mandatory or discretionary. Usually, when the driver must need to change from current lane to desired lane, then this type of changing is called mandatory lane-changing. Other type of changing is called discretionary lane-changing, when driver want to improve the vehicle speed conditions or comfortable journey or over taking to lead vehicle or passing heavy vehicle or avoiding the traffic conditions near off-ramp or on-ramp or road condition is not good, it is not must needed to change the lane, then driver decide the discretionary LC $[32,33]$. For mandatory LC, driver is more aggressive who sometimes avoid gap acceptance and forces the other drivers [34]. In this research, Researchers have analyzed these two types of LC for car. After taking the decision of drivers (discretionary and mandatory), Driver must calculate the gap at target lane to ego vehicle and lead vehicle. Then the ego vehicle goes by dynamical strategies like as position, velocity, acceleration and deceleration for avoid collision to the surrounding vehicles. Materially, the proposed procedure can also be realistic to heavy vehicles or car discretionary and mandatory LC vehicles by defining them as ego vehicles [27].

In this section, researchers have discussed LCM by flowchart then analyzed the methodology of RKHS, non-parametric regression fitted model. This model is generated the LC beginning and finishing points by using NGSIM data, but researchers couldn't explain the LC behavior of Chinese freeway data. The LC decision algorithm is discussed as follows by flowchart in Figure 3 [35].

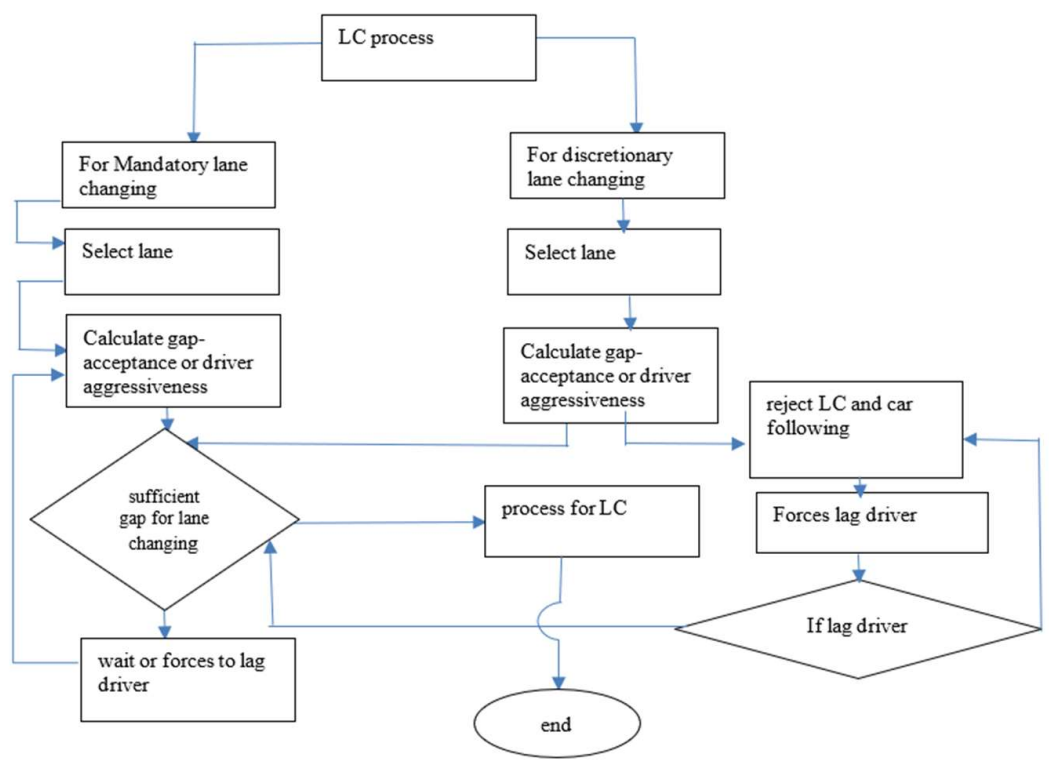

Figure 3. LC behavior flowchart

\subsubsection{LC method for behavior generator in RKHS}

A real trajectory is plainly designated by Gaussian-radial-basis-functions, by using RKHS of functional gradient to improve cost functional to static difficulties or direct high dimensional arm to voids [28]. Researchers monitor previous design, however by using functional-gradient the optimal solution is obtained, our reviewed method depends on RKHS with non-parametric-regression and previous dataset to evaluate continuous values as the start or end points. Reproducing-Kernel-Hilbert-Space H holds families of smooth-functions which are distinct by Mercer-Kernel. Again Mercer-Kernel is continuous mapping [29].

$$
K: X \times X \rightarrow I R, \text { i.e. }, K(x, y)=<p, q>H, \text { where } p=K_{x}, q=K_{y}, p, q \in H
$$

A linear-combination of this kernel, $p()=.\sum \alpha_{i} K_{x i}($.$) with reproducing property p(x)=<p, K_{x}>$ is a function in $\mathrm{H}$ [29]. Another function $F: \Psi \rightarrow B$ where $X \varepsilon \Psi$ and $b \varepsilon B$ is behavior generator. The coordinate space $\Psi$ is included surrounded group of cars position of real trajectory where $X \stackrel{\text { def }}{=}$ $\left\{x_{i}\right\}_{1}^{N}, x_{i} \varepsilon I R^{T}$. T is length of previous position. The output vector is $\Omega=\{X\}_{i}^{N}$ as practice set. The kernel is a 
matrix-valued-function $\Psi \times \Psi \rightarrow I R^{D \times D}$ where $\mathrm{D}$ is dimension of the problem, where dimension is 2 in this research for finding the beginning and finishing points of the LC behavior. Where

$$
K(X, U)=\left[\begin{array}{ccc}
K(X, U)_{1,1} & \cdots & K(X, U)_{1, D} \\
\cdots & \cdots & \cdots \\
K(X, U)_{D, 1} & \cdots & K(X, U)_{D, D}
\end{array}\right]
$$

The linear-combination of the kernel as following is inner-product-space [29].

$$
G(X)=\sum_{1}^{N} K\left(X_{i}, X\right) \cdot \alpha_{j}, \alpha_{j} \varepsilon I R^{D}
$$

\subsubsection{Non-parametric-regression for beginning-finishing points of $\mathrm{LC}$ behavior}

The regularized empirical error is minimized by the below approximation function [29].

$$
\hat{p}=\underset{p \varepsilon H}{\arg \min } \sum_{1}^{N}\left(b_{i}-p\left(X_{i}\right)\right)^{2}+\mu L(p)
$$

Where $X_{i}$ and $b_{i}$ are practice input and output of behavior and $L(p)$ is the penalty terms. The solution of the coefficient term is solved by the following equation.

$$
\alpha=(K(X, U)+\mu N I)^{-1} b
$$

From the above equation, the optimization behavior generator is following equation.

$$
\widehat{b}=K^{*}(K+\mu I)^{-1} b
$$

Where $b \stackrel{\text { def }}{=}\left\{b_{i}\right\}_{1}^{N}$, collection of practice data set and $K^{*}$, new kernel from previous equations. The distance formula of multi-quadratic kernel.

$$
K\left(X_{1}, X_{2}\right)=\frac{1}{\sqrt{\left\|X_{1}-X_{2}\right\|^{2}+c}} \text { Where } \mathrm{c}>0
$$

Where $X_{1}, X_{2} \varepsilon \Psi$ are matrices,

The Hilbert-Schmit norm give the kernel value by,

$$
\left\|\left(X_{1}-X_{2}\right)=W\right\|_{p}=\sqrt{\operatorname{tr}\left(W^{T} \cdot W\right)}
$$

A trace function is represented by $\operatorname{tr}($.$) ,$

$$
\|W\|_{p}=\|W\|_{2}=\sqrt{\mu_{\max }\left(W^{T} \cdot W\right)}
$$

A spectral norm and Frobenenius norm are consider a singular value of matrix.

$$
\sqrt{\operatorname{tr}\left(W^{T} \cdot W\right)}=\sqrt{\sum \vartheta_{i}^{2}}
$$

Matrix W has a singular value is $\vartheta_{i}$

$$
\sqrt{\mu_{\max }\left(W^{T} \cdot W\right)}=\max _{i} \vartheta_{i}
$$

A main difference of matrices is calculated by the singular values which takes from real trajectories.

\subsubsection{LC algorithm}

By applying RKHS, non-parametric regression fitted line of begging and finishing points from real trajectory data set is given as follows in Figure 4 [29]. 


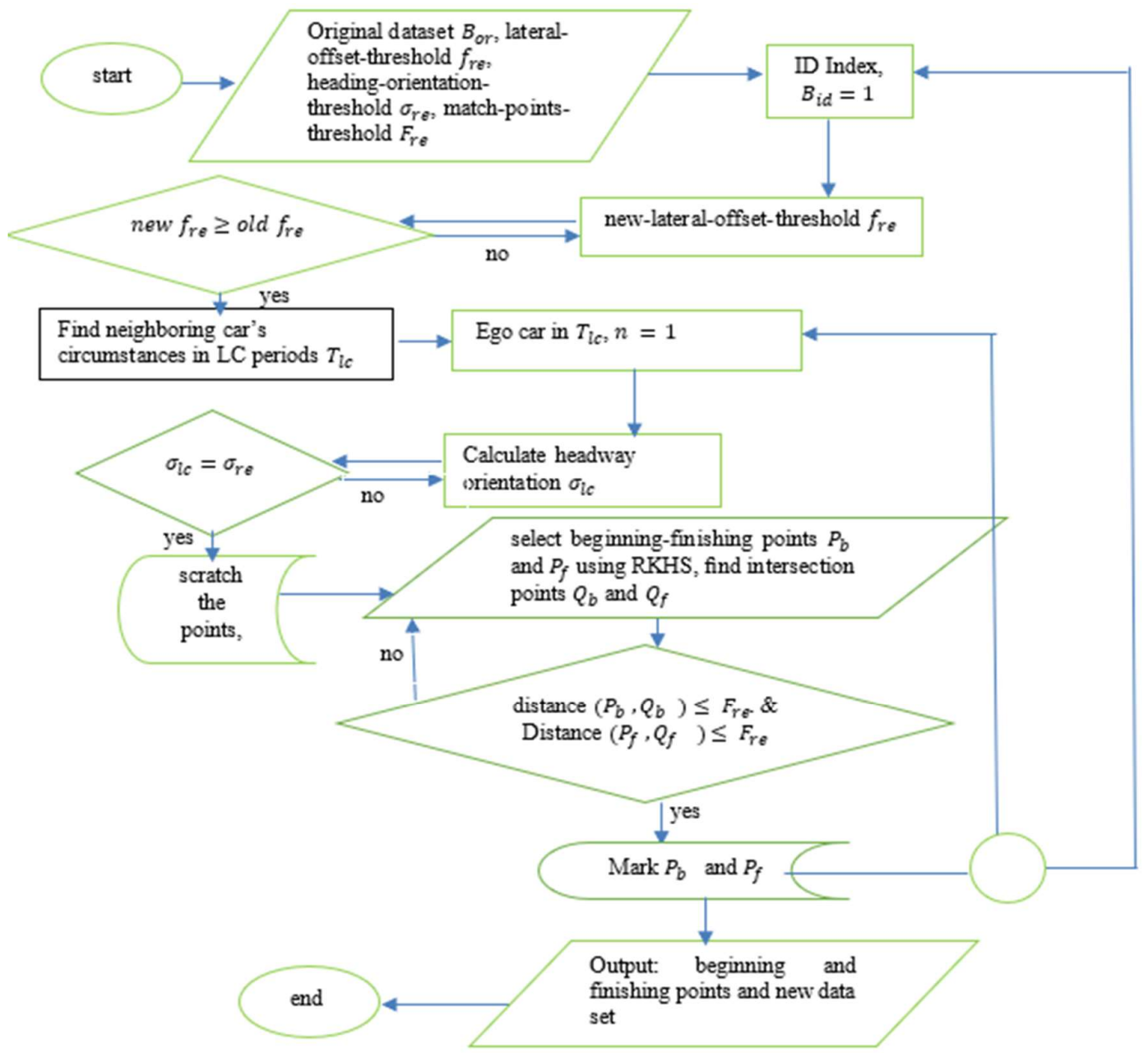

Figure 4. LC dynamics flowchart

\section{RESULT AND DISCUSSION}

\subsection{Data collection and procedure}

The NGSIM data are collected from US-101 highway with on-ramp and off-ramp by high proficiency video camera from 50 meters high and the length of the lane is 500 meters at different time and by simulation software, this data is created on the excel sheet including car position, velocity, acceleration, gap to front car, time headway to front car, lane changes scenario and so on which taken from popular website (www.ngsim.fhwa.dot.gov). Again, Guo, et al. [36], collected the Chinese data from Beijing forth ring road, west bridge, west bound, urban expressways with on-ramp (downstream) and off-ramp (upstream) at 9.05 am to 9.35 am in November 03, 2008 sunny day and section length 100 meters [36]. This data has car-id, time, position, speed, time-headway, gap and acceleration. For compare this data with US-101 data, researchers have created the data like US-101 such as only two times positions of data are created to one hundred times position data for every column by using position-speed-acceleration equations. After that, since time is different than US-101, researchers have assumed same time that is the two types of data are at same time. Again, for LC analysis, data is required with longitudinal position and lateral position, but in Chinese data has no these types of column, for these, researchers couldn't able to compare the LC scenario with US-101 data. Only LC analysis have been discussed for US-101 data.

\subsection{CFM}

The following parameters values are given by the GA calibration of IDM CFM. Five types parameter as maximum acceleration, minimum deceleration, freeway car speed, minimum time headway and stopping distance for US-101 data and Chinese data is many far different time headway, more different at 
minimum deceleration and maximum acceleration and near is free speed and stopping distance from the Table 1. The rang of the parameters as follows and assumed parameters are maximum acceleration, $a=$ $1.4 \mathrm{~m} / \mathrm{s}^{2}$, minimum deceleration, $b=2.0 \mathrm{~m} / \mathrm{s}^{2}$, desired speed, $v_{0}=30 \mathrm{~m} / \mathrm{s}$, desired headway, $T=1.5 \mathrm{~s}$, jam minimum distance, $s_{0}=2 \mathrm{~m}$ [21]. The CF parameters as maximum acceleration, minimum deceleration, free speed, minimum headway and stopping distance percentages of Chinese data are $74.71 \%, 79.95 \%$, $66.57 \%, 0.018 \%$ and $65.65 \%$ respectively of NGSIM data. Here, the minimum headway is near zero of Chinese data and acceleration and deceleration are near to maximum. So, stopping distance of Chinese data is far than NGSIM data. From these discussion, US-101 data is safer and more comfortable than Chinese data.

Table 1. Parameter values for US-101 data and Chinese data

\begin{tabular}{lccccc}
\hline & Max. Accel. & Min. Decel. & Free speed & Min. headway & Stopping dist. \\
\hline IDM parameter range & {$[1,3]$} & {$[1,4]$} & {$[10,30]$} & {$[0,3]$} & {$[1,10]$} \\
US-101 & 1.001959 & 1.002857 & 15.06155 & 1.224402 & 5.231103 \\
Chinese data & 2.96130 & 3.99999 & 29.9999 & 0.00023 & 9.99825 \\
Percentage of Chinese data & $74.71 \%$ & $79.95 \%$ & $66.57 \%$ & $0.018 \%$ & $65.65 \%$ \\
\hline
\end{tabular}

\subsubsection{CFM figure explanation}

Figure 5 and Figure 6 represent driver CF behavior position of US-101 data and Chinese data respectively that group of cars are going so fast but NGSIM car suddenly slow. Figure 7 and Figure 8 represent the observation value of real trajectory and calibration value by GA of gap and speed difference on time. For NGSIM data, calibration data is moving very nearly with observation data for gap and speed difference more than Chinese data. From $700 \mathrm{~s}$ to $730 \mathrm{~s}$ the gap of Chinese data is more than $100 \mathrm{~m}$ and $700 s$ to $710 s$, the speed difference is more than $30 \mathrm{~m} / \mathrm{s}$. From Figure 9 and Figure 10, the objective function SSE values very early decrease of NGSIM data than Chinese data. And the Chinese data objective values of GA are starting from $2.7 \times 10^{6}$ to $1.9 \times 10^{6}$ but the NGSIM data objective values are starting from near 850 to near 300 that are extremely difference. Figure 11 and Figure 12 are the fitness value of these data by GA are represented that NGSIM data fitness value is low than Chinese data. At GA, best fitness values and mean fitness values of NGSIM data is more near than Chinese data. From the above discussion, NGSIM data is nicely simulated than Chinese data is claimed by authors.

\subsection{Experimental result of LCM}

In the NGSIM real trajectory data has eighteen columns including local $\mathrm{X}$ and local $\mathrm{Y}$ which represent the longitudinal and lateral position respectively and every car has lane id, velocity, acceleration, gap, time headway and time are very necessary for LC behavior analysis. Although in the Chinese data has only six columns as car id, car velocity, acceleration, longitudinal position, frame id, gap and time headway and it has not including local $\mathrm{X}$ and local $\mathrm{Y}$ that is very necessary for LC behavior analysis. Every group of car as front car, ego car, rear car, front car at target lane, rear car at target lane at left side and right side in NGSIM data have car id, car velocity, acceleration, longitudinal position, frame id, gap and time headway with recorded data within 10 seconds. Front car follows the surrounding car's dynamics for understanding LC maneuver. For start the lane-changing, front car applies the maneuver. Sections of real trajectories data from all group of cars before the front car starts rotating towards near target lanes are reserved input data as $\mathrm{X}$. For LC behavior, parameter value $\alpha$ has been obtained from given value $b$ by above equation. After knowing parameter value $\alpha$ has been applied to another equation for finding new response kernel $\mathrm{K}^{*}$ is measured using the data set. And finally, the estimation value $\hat{b}$ is obtained. Figure 13 is shown LC start beginning and finishing points, dots are indicating LC time of car and black line is indicating the before and after LC time which is maneuver for driver by given above formulation, Figure 14 is represented the longitudinal and lateral car position maneuver of ego car for different group, where first, second and fourth group cars are blue, red and orange color respectively are changing from middle lane to right lane (first line) and only purple color car of third group is changing from third lane to middle lane. Figure 15 has been shown the LC position of a group of car, since time is not included in the graph, so the position of lateral and longitudinal is interchanging. If time is included in the graph, then it will be three-dimensional graph. 


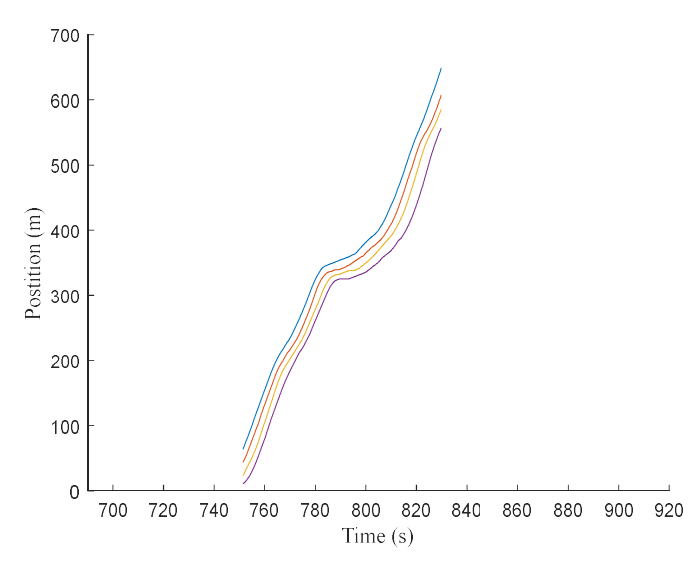

Figure 5. Car position for US-101
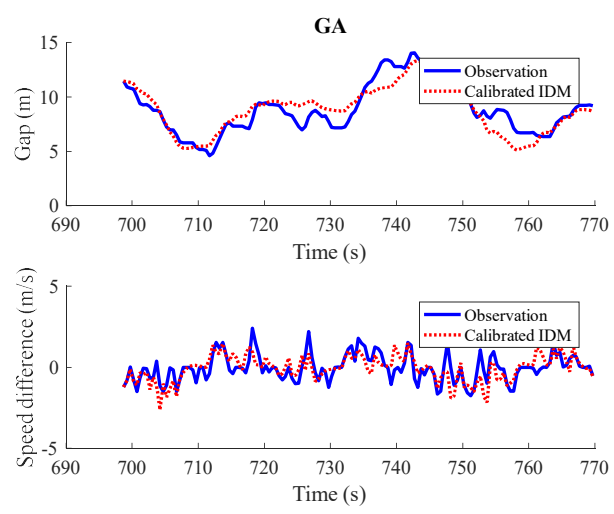

Figure 7. Calibration with observation by GA for US-101

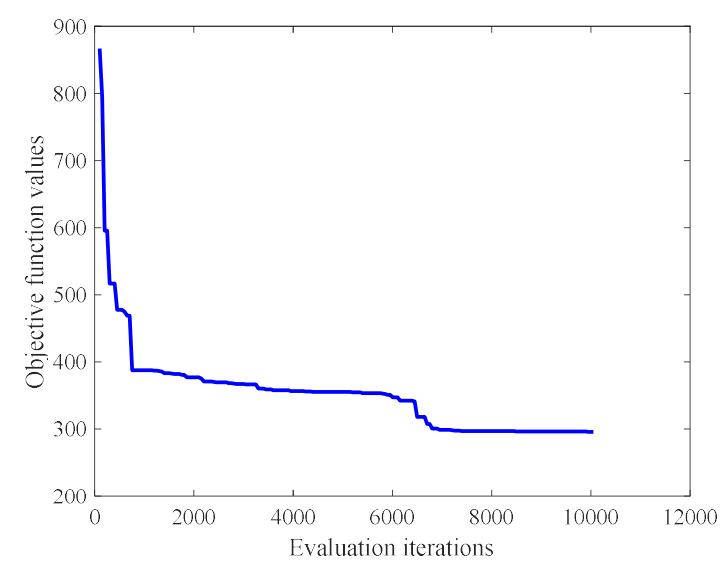

Figure 9. Objective function value of GA for US-101 data

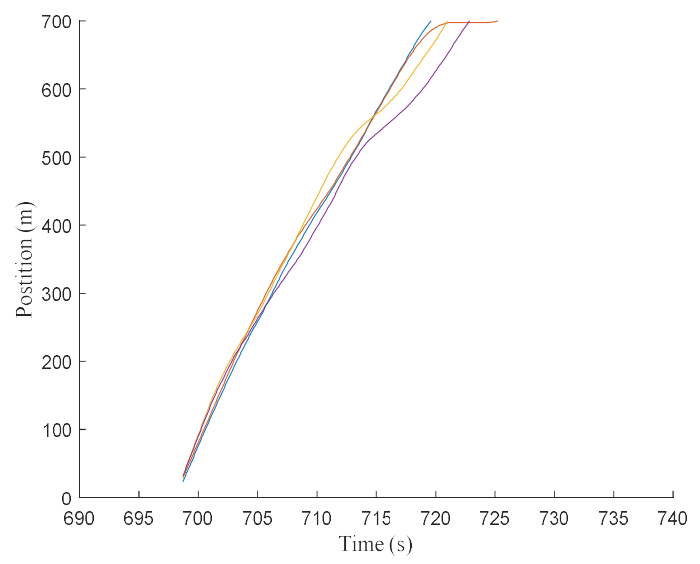

Figure 6. Car position for Chinese freeway
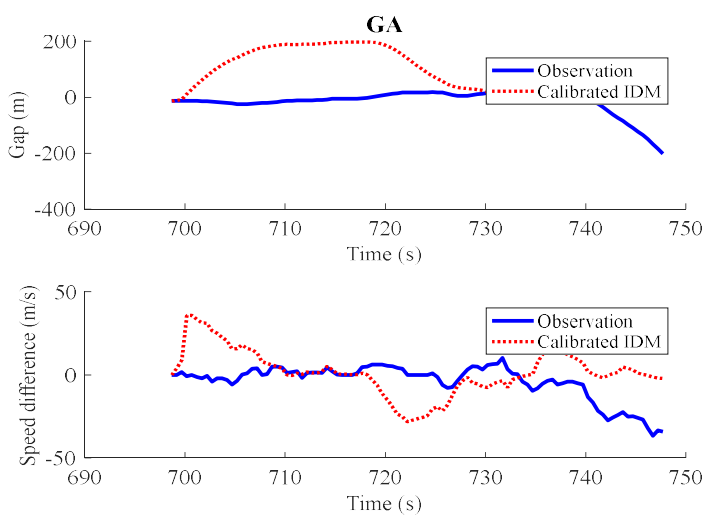

Figure8. Calibration with observation by GA for Chinese freeway

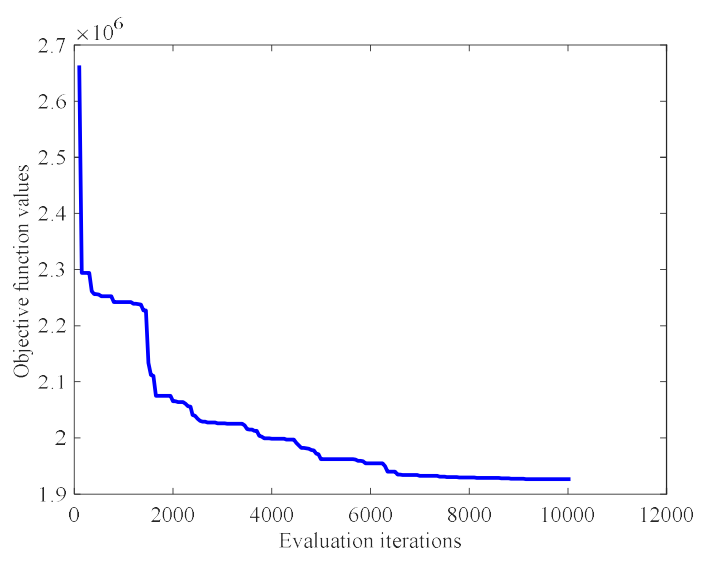

Figure 10. Objective function value of GA for Chinese data 


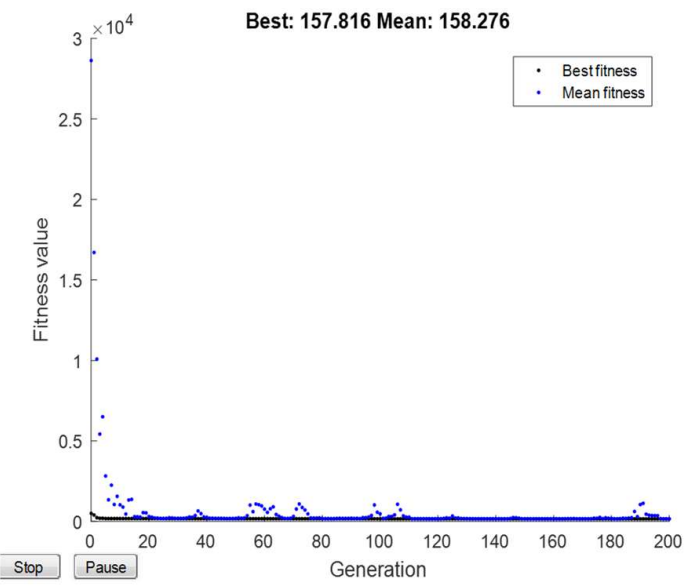

Figure 11. Fitness values of GA US-101

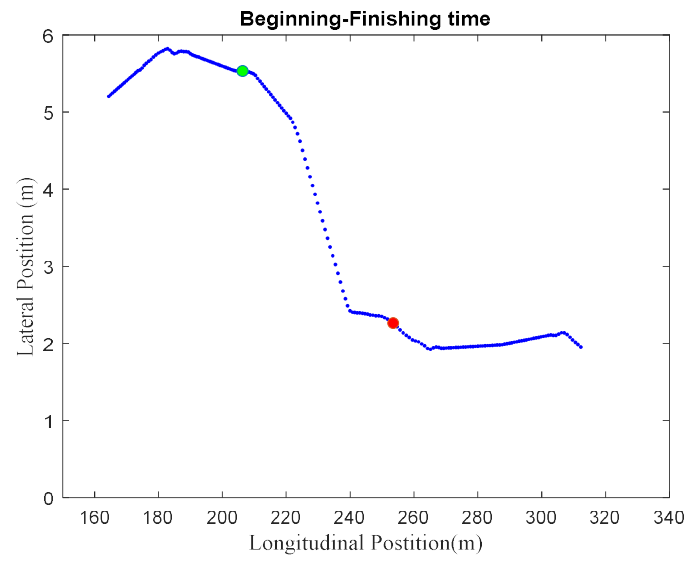

Figure 13. LC beginning and finishing points of ego car

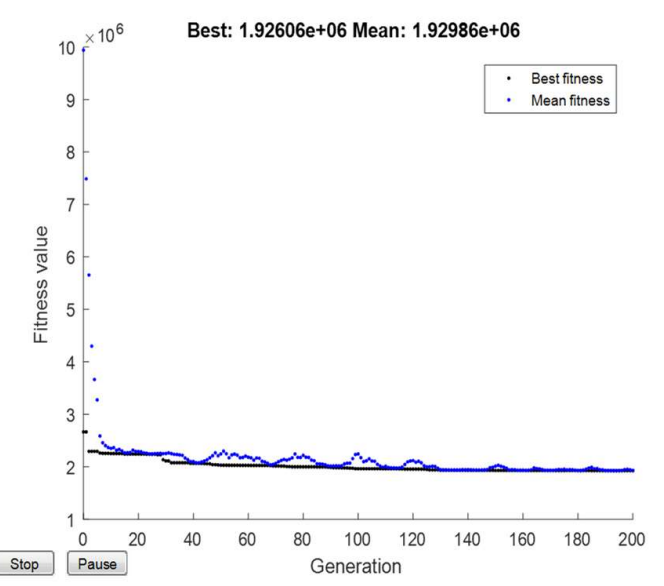

Figure 12. Fitness values of GA for Chinese data

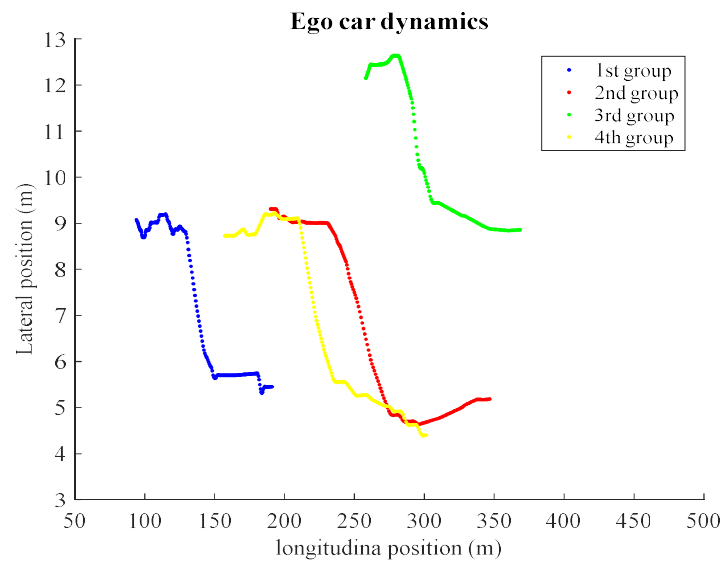

Figure 14. LC position of ego car of different group

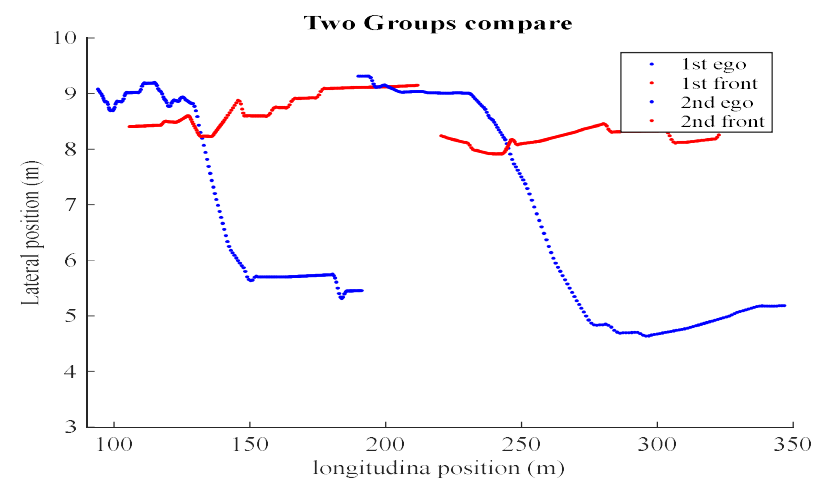

Figure 15. LC position of front car and front car of same group

\section{CONCLUSION}

The research elementary focus is to compare driver behavior evaluation contextual of different state of US-101 and Chinese freeway road by using many efficient IDM of CF and LC car dynamics. This CF simulation model is calibrated by well-known GA using SSE objective function. The US-101 driver behavior

Car following and lane changing behavior using NGSIM and China data (Md. Mijanoor Rahman) 
real trajectory data is well simulated then the Chinese freeway data because NGSIM CF calibration data and simulation data is more likely such as input CF parameter values and output values is very near than Chinese data. The ego car is following the front car according to IDM parameter values as position, gap, headway, velocity, comfortable Acceleration and deceleration if necessary, otherwise driver is changing lane for mandatory or discretionary LC behavior by calculating the safe gap and beginning-finishing points of this time. Based on aforementioned models, driver must understand their behavior and can act for driving car at congested or freeway traffic network. Then, these models have given the result to the driver about their driving system that is, when the driver follow the car and when the driver change the lane about the maneuver for going fast and comfortable. When driver can understand the lane changing time and acceptable gap then car collisions or other crashes are not happened. This research has very helpful for acting to the road safety by analyzing the car-following and lane changing decision of the drivers. After analyzing, this work has been given a strong suggestion and rule to the road user. If driver can understand their behavior about road traffic system by CF and LC, then traffic safety and road crashes will be decreased. Then driver can go very fast avoid accident and the traffic flow has been increased. By analyzed the different scenario, traffic network will bestow the generalized CF or LC behavior which will be the life safe and comfortable journey of human drivers. By properly extracted data for future work, CF calibration parameters and LC behavior can give the rule for road safety, comfortable and time-consuming journey.

\section{REFERENCES}

[1] S., Ma, et al., "Road traffic injury in China: a review of national data sources", Traffic injury prevention, vol. 13(sup1), pp.57-63, 2012.

[2] H. Hao, et al., "Natural gas as vehicle fuel in China: A review." Renewable and sustainable energy reviews, vol. 62, pp. 521-533, 2016.

[3] D. Nepelski and D. P. Giuditta, "International technology sourcing between a developing country and the rest of the world. A case study of China," Technovation, vol. 35, pp. 12-21, 2015.

[4] Y. Li and D.Sun, "Microscopic car-following model for the traffic flow: the state of the art" Journal of Control Theory and Applications, vol. 10(2), pp. 133-143, 2012.

[5] J. Zheng, et al., "Predicting driver's Lane changing decisions using a neural network model," 2013 IEEE Simulation Modelling Practice and Theory, vol. 42, pp. 73-83, 253-1258, 2014.

[6] H. N. Koutsopoulos and H. Farah, "Latent class model for car following behavior", Transp. Res. Part B: Methodol., vol. 49, pp. 563-578, 2012.

[7] J. (Ed.) Barceló, "Fundamentals of Traffic Simulation," Springer, 2010.

[8] D. Chen, et al., "A behavioral car following model that captures traffic oscillations. Transp," Res. Part B: Methodol., vol. 46(6), pp. 744-761, 2012.

[9] Kesting, M. Treiber, "Calibrating car-following models by using trajectory data methodological study," Transp. Res. Rec., vol. 2088, pp.148-156, 2008.

[10] V. Punzo, et al., "Can results of car following model calibration based on trajectory data be trusted?," Transp. Res. Rec., vol. 2315, pp. 11-24, 2012

[11] V. Punzo and F. Simonelli, "Analysis and comparison of microscopic traffic flow models with real traffic microscopic data", Transp. Res. Rec., vol 1934, pp.53-63, 2005.

[12] S. Ossen and S.P. Hoogendoorn, "Reliability of parameter values estimated using trajectory observations", Transp. Res. Rec., vol. 2124, pp. 36-44, 2009.

[13] B. Ciuffo, et al., "Thirty years of Gipps' car-following model," Transp. Res. Rec., vol. 2315, pp.89-99, 2012.

[14] E. Chung, A. G. (Eds.) Dumont, Transportation Simulation: Beyond Traditional Approaches. CRC Press, 2009.

[15] E. Brockfeld, et al., "Calibration and validation of microscopic models of traffic flow," Transportation Research Record: Journal of the Transportation Research Board, vol. 1934, pp. 179-187, 2005.

[16] S. Ossen and S.P. Hoogendoorn, "Validity of trajectory-based calibration approach of car following models in presence of measurement errors", Transp.Res. Rec, vol. 2088, pp. 117-125, 2008.

[17] P. Wagner, "Analyzing fluctuation in car following," Transp. Res. Part B: Methodol., vol. 46, pp. 1384-1392, 2012.

[18] Paz, et al., "Calibration of traffic flow models using a memetic algorithm, "Transportation Research Part C: Emerging Technologies, vol. 55, pp. 432-443, 2015.

[19] V. Punzo and A. Tripodi, "Steady-state solutions and multiclass calibration of Gipps microscopic traffic flow model", Transp. Res. Rec., vol. 1999, pp. 104-114, 2007.

[20] L. Li, et al., "A global optimization algorithm for trajectory data-based car following model calibration," Transportation Research Part C: Emerging Technologies, vol. 68, pp. 311-332, 2016.

[21] J.A. Nelder and R. Mead, "A simplex method for function minimization," Comput. J., vol. 7, pp. 308-313, 1965.

[22] P. G. Gipps, "A behavioural car following model for computer simulation," Transp. Res. B: Methodol., vol. 15, pp. 105-111, 1981.

[23] H. Rakha and Y. Gao, "Calibration of steady-state car following models using macroscopic loop detector data. In: Proceedings of 75 Years of the Fundamental Diagram for Traffic Flow Theory", Greenshields Symposium, pp. 178-198, 2011.

Int. J. of Adv. in Appl. Sci. Vol. 8, No. 1, March 2019: $14-25$ 
[24] B. Ciuffo and V. Punzo, "No Free Lunch theorems applied to the calibration of traffic simulation models," IEEE Trans. Intell. Transp. Syst., vol. 15(2), 5, pp. 53-562, 2014.

[25] J.C. Lagarias, et al., "Convergence properties of the Nelder-Mead simplex method in low dimensions", SIAM J. Optim, vol. 9(1), pp. 112-147, 1998.

[26] J. Nilsson and J. Sjöberg, "Strategic decision making for automated driving on two-lane, one way roads using model predictive control," in Intelligent Vehicles Symposium (IV), 2013 IEEE, pp. 1253-1258, 2013.

[27] Y. Hongtao, et al., "A human-like game theory-based controller for automatic lane changing," Transportation Research Part C: Emerging Technologies, vol. 88, pp. 140-158, 2018.

[28] Z. Marinho, et al., "Functional gradient motion planning in Reproducing Kernel Hilbert Spaces", arXiv preprint arXiv: $1601.03648,2016$.

[29] C. Dong, Y, Zhang, and J. M. Dolan, "Lane-change social behavior generator for autonomous driving car by nonparametric regression in Reproducing Kernel Hilbert Space," in Intelligent Robots and Systems (IROS), 2017 IEEE/RSJ International Conference, pp. 4489-4494, 2017.

[30] M. Rahman, et al., "Evaluation of Driver car following Behavior Models for Cooperative Adaptive Cruise Control Systems," Transportation Research Record: Journal of the Transportation Research Board, vol. 2622, pp.84-95, 2017.

[31] B. Ciuffo and V. Punzo, "Verification of traffic micro-simulation model calibration procedures: Analysis of goodness-of-fit measures," in 89th Annual Meeting of the Transportation Research Board, Washington, DC, 2010.

[32] P. G. Gipps, "A model for the structure of lane changing decisions," Transportation Research Part B: Methodological 20, vol. 5, pp. 403-414, 1986.

[33] T. Toledo, et al., "LCM with explicit target lane choice," Transportation Research Record: Journal of the Transportation Research Board, vol. 1934, pp.157-165, 2005.

[34] P. Zhao, et al., "Analysis of mandatory and discretionary lane change behaviors for heavy trucks," arXiv preprint arXiv:1707.09411, 2017.

[35] X. Li and J.Q. Sun, "Studies of vehicle lane changing dynamics and its effect on traffic efficiency, safety and environmental impact," Physical A: Statistical Mechanics and its Applications, vol. 467, pp.41-58, 2017.

[36] M. Guo, et al., "Empirical study of LC behavior on three Chinese freeways," PloS one, vol. 13(1), e0191466, 2018.

\section{BIOGRAPHIES OF AUTHORS}

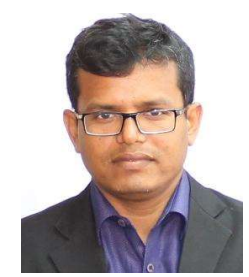

Md. Mijanoor Rahman, current Ph.D student at school of mathematical science in Universiti Sains Malaysia (USM). Now he is working on Traffic flow network by using car following with some calibration and lane changing model. Currently he is an Assistant professor at mathematics department in Mawlana Bhashani Science and Technology, Tangail, Bangladesh. He has total Eight years teaching experience at different university. He completed his master's degree and bachelor's degree at mathematics department in Chittagong University, Bangladesh. He has previous eight publications in different journals. The authority of Mawlana Bhashani Science and Technology have given the Study leave with pay salary for doing his $\mathrm{PhD}$ with research.

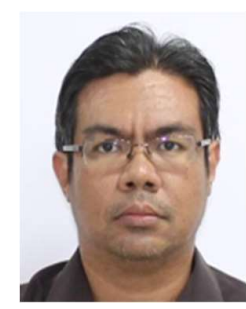

Dr. Mohd Tahir Ismail is Associate Professor and researcher in the School of Mathematical Sciences, Universiti Sains Malaysia (USM). The research area is on financial time series. Particularly he is keen in the modeling of structural breaks and analyses the economics issues. As of now, he has published more than 100 publications in reviewed journals and proceedings (some of them are listed in ISI, Scopus, Zentralblatt and other indices). His research grand is the extension of indicator saturation approach in GARCH Model to detect structural break and outliers: Empirical evidence in Malaysia Shariah-compliant Indices, FRGS, 2017-2019. Many trainings \& conferences have been participated as International Seminar on Mathematics in Industry (ISMI2017), International Conference on Forecasting Economic and Financial Systems, Bodies \& Professional is Malaysian Mathematical Sciences Society (PERSAMA), Malaysia Institute of Statistics (ISM).

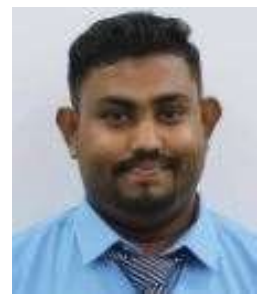

Dr Majid Khan joined the School of Mathematical Sciences, USM, as a lecturer (Operational Research) in May 2017. He is a researcher and appointed fellow working in the field of seaweed cultivation, solar drying systems, processing, modelling and simulation. His research uses application of Iot, big data and simulation methods to improve model predictions of moisture losses during drying in control and uncontrolled environment. He is also interested in modelling the problems in engineering and other biological systems such as tissue culture and aquamarine. He uses the techniques from statistical theory, approach and existing application tools to develop mathematical model and finally to transform the model in industry application and to answer a range of inspired questions. 